\title{
Simultaneous Triple Primary Neoplasms in the Gastrointestinal Tract: A Rare Case Report
} \author{
Xiujun Cai*1,2 \\ ${ }^{1}$ Department of General Surgery, China \\ ${ }^{2}$ Key Laboratory of Laparoscopic Technology of Zhejiang Province, China \\ ${ }^{3}$ Department of Gastrointestinal Surgery, China \\ ${ }^{4}$ Department of Anesthesiology, China
}

Xingyu Lv ${ }^{1,2}$, Guangyi Jiang ${ }^{1,2}$, Qiang Hong ${ }^{3}$, Yifan Wang ${ }^{1,2}$, Xinjie Zhang ${ }^{1,2}$, Jisheng Lv ${ }^{4}$ and

*Corresponding author: Xiujun Cai, Department of General Surgery, Sir Run Run Shaw Hospital, School of Medicine, Zhejiang

University, Hangzhou, 310016, Zhejiang Province, China

\section{ARTICLE INFO}

Received: 㗀 January 30, 2019

Published: 豐 February 07, 2019

Citation: Xingyu L, Guangyi J, Qiang H, Yifan W, Xinjie Z, Jisheng L, Xiujun C. Highlights on the Selective CDK4/6 Inhibitors. Biomed J Sci \& Tech Res 14(2)-2019. BJSTR. MS.ID.002517.

Keywords: Case Report; Multiple Primary Neoplasms; Primary Colon Cancer

Abbreviations: MPNs: Multiple Primary Neoplasms; CT: Computed Tomography; CEA: Carcinoembryonic Antigen

\section{ABSTRACT}

Background: Multiple primary neoplasms (MPNs) are of rare occurrence. The majority of MPNs are double primary neoplasms while triple and quadruple primary neoplasms are extremely rare. The clinical diagnosis of MPNs relies on pathological examination and pathogenesis is still unclear.

Case Presentation: Here, we report a rare case of a 78-year-old woman diagnosed with simultaneous triple primary neoplasms in the ascending colon, the transverse colon and the appendix. The patient presented with lower abdominal pain in the right upper quadrant of 2 months duration. The serum carcinoembryonic antigen (CEA) level (185.83 $\mathrm{ug} / \mathrm{L}$ ) was significantly higher than the normal range (0-5 ug/L). An abdominal computed tomography (CT) scan with intravenous contrast scan showed multiple soft tissue masses in the hepatic flexure of the ascending colon and transverse colon, and a $2.8 \times 2.8 \mathrm{~cm}$ soft tissue mass in the right ileocecal appendix. She underwent a laparoscopic radical extended right hemicolectomy. The patient has been seen in follow-up every 3 months, and there has been no recurrence after 16-months. We explore the etiology and discuss the treatment and prognosis of MPNs.

Conclusions: Simultaneous triple primary neoplasms are uncommon cases. Image examination and pathological evaluation are pivotal for diagnosis. Although the pathogenesis of MPNs is unclear, we believe that poor dietary habits were the largest contributor in this case. Surgery-based comprehensive therapy is the recommended therapy for simultaneous multiple primary neoplasms.

\section{Background}

Multiple primary neoplasms (MPNs) were not recognized until the 1890s [1]. MPNs were first defined by Warren and Gates in 1932 [2]. The diagnostic criteria for MPNs are as follows:

a) at least two neoplasms are detected in an individual patient;

b) each neoplasm has its identified pathology;

c) the possibility of one being a metastasis of the other must be ruled out.
In 1961, Moertel [3] proposed that if MPNs are diagnosed within 6 months, they are simultaneous MPNs; if MPNs are diagnosed with a time interval more than 6 months, they are metachronous MPNs. Today, the diagnosis of MPNs has become more precise due to the development of imaging equipment and pathology examinations [4]. The incidence of MPNs in cancer patients is estimated to be $0.5 \%$ to $10 \%$ [5]. The majority of MPNs are metachronous double primary neoplasms, while simultaneous triple and quadruple primary neoplasms are much rarer [6]. In this report, we provide 
a rare case of simultaneous triple primary adenocarcinomas of the ascending colon, transverse colon, and appendix.

\section{Case Presentation}

A 78-year-old female presented with lower abdominal pain in the right upper quadrant of 2 months duration without remarkable previous disease histories. The abdominal pain was paroxysmal with nausea and vomiting and the pain relieved spontaneously. The serum carcinoembryonic antigen (CEA) level (185.83 ug/L) was significantly higher than the normal range $(0-5 \mathrm{ug} / \mathrm{L})$. No member in her family was ever diagnosed with neoplasm. The patient reported a $5 \mathrm{~kg}$ weight loss in the past three months. Physical examination revealed pain on palpation of the right upper abdomen. An abdominal computed tomography (CT) scan with intravenous contrast scan (Figure 1) showed multiple soft tissue masses in the hepatic flexure of the ascending colon and transverse colon, and a $2.8 \times 2.8 \mathrm{~cm}$ soft tissue mass in the right ileocecal appendix. A large mass was found in her transverse colon by enteroscopic examination (Figure 2). Colonoscopy and biopsy revealed a tubulovillous adenoma in the transverse colon (Figure 3).

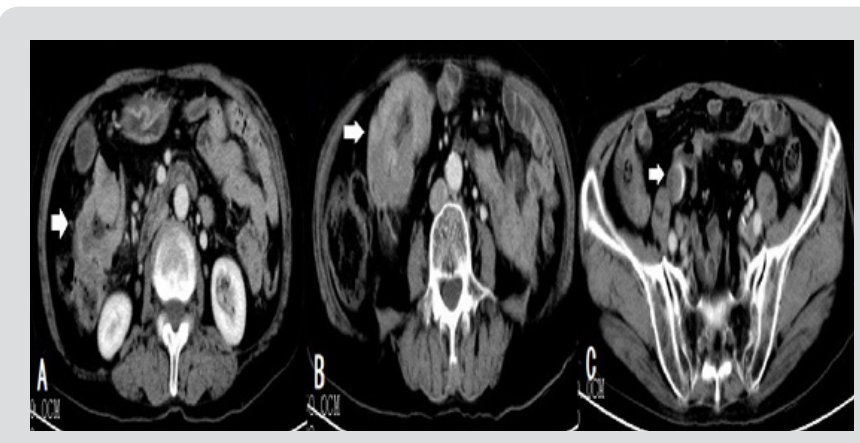

Figure 1: Abdominal contrast-enhanced CT scan CT reveals marked bowel-wall thickening of the ascending colon (A) and the transverse colon (B), and the appendix (C) is markedly enlarged.

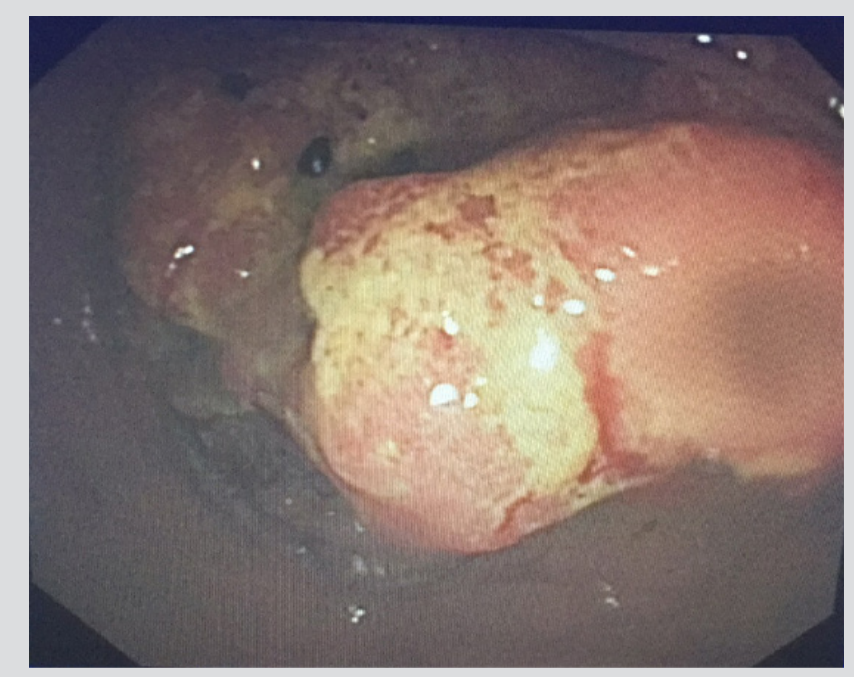

Figure 2: Enteroscopy shows a large mass in the transverse colon.

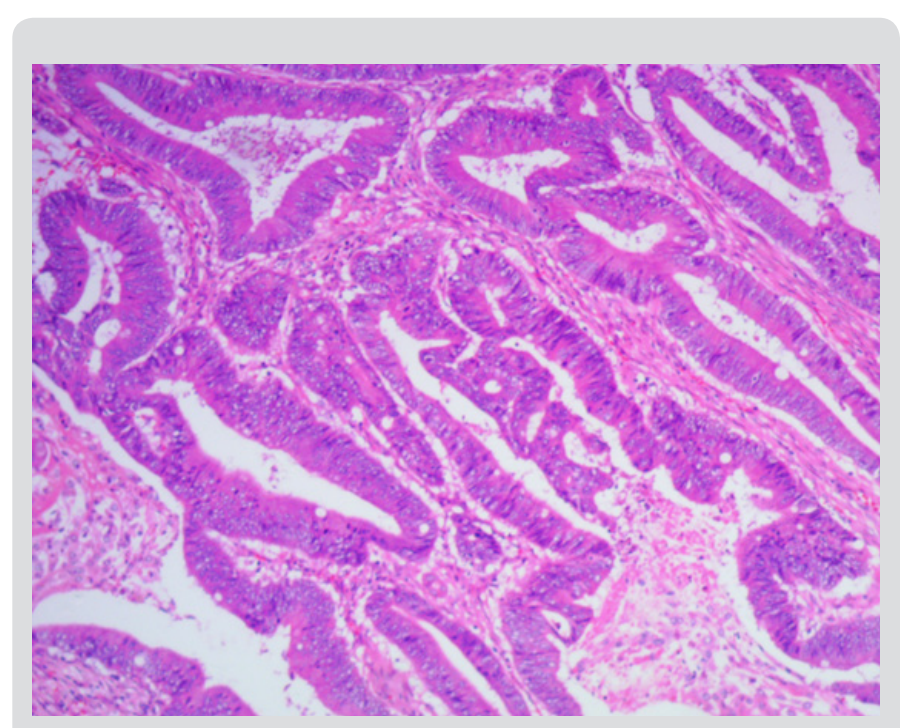

Figure 3: Histopathology of colonoscopy samples shows villous-tubular adenomas in the transverse colon. (magnification $\times 200)$.

After multidisciplinary discussion, a laparoscopic radical extended right hemicolectomy was performed. Surgical findings (Figure 4) showed a $6 \times 5 \mathrm{~cm}$ mass in the hepatic flexure of the ascending colon and a $4 \times 4 \mathrm{~cm}$ mass in the hepatic flexure of the transverse colon. The two masses, leading to incomplete intestinal obstruction, were $4 \mathrm{~cm}$ apart. Postoperative histopathology of the specimen from the ascending colon (Figure 5A) revealed a moderately differentiated adenocarcinoma infiltrating the intestinal wall. Moreover, the mass from the appendix was diagnosed as a low-grade appendiceal mucinous neoplasm (Figure 5B). The postoperative course was uneventful. The patient refused to receive postoperative chemotherapy, radiotherapy, immunotherapy or targeted therapy. The patient has been seen in follow-up every 3 months, and there has been no recurrence after 16-months.

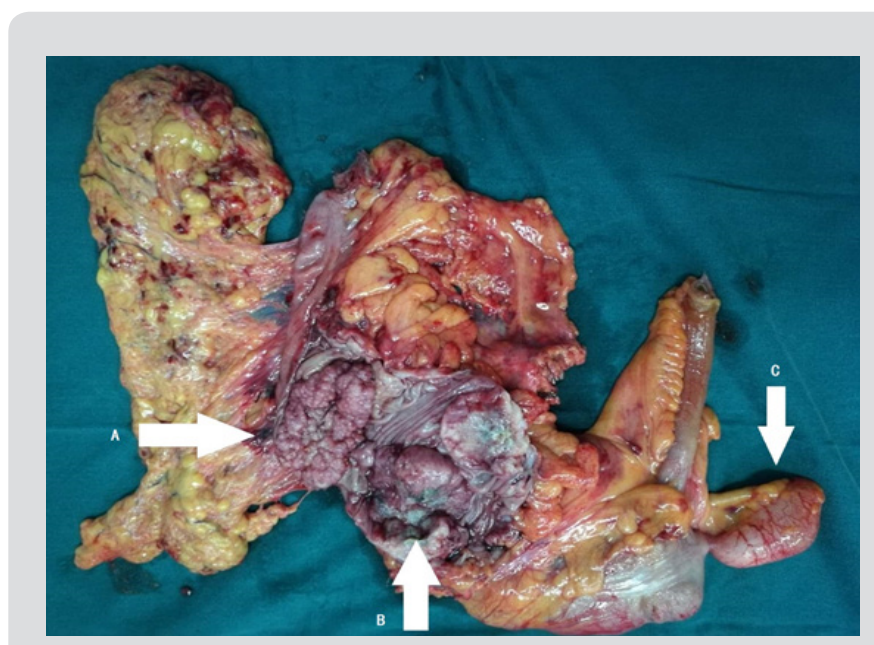

Figure 4: The resected specimens. Specimens from the hepatic flexure of the transverse colon (A), the ascending colon (B), and the appendix (C). 


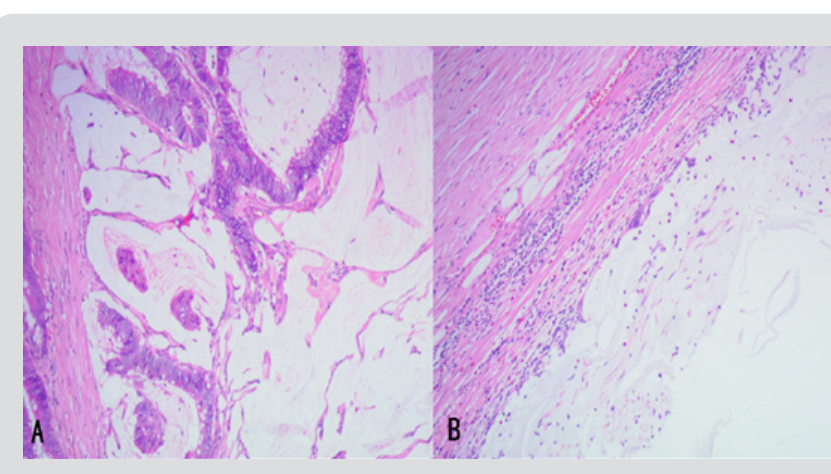

Figure 5: Surgical pathology. Two infiltrating, moderately differentiated adenocarcinomas in the ascending colon (A) and a low-grade appendiceal mucinous neoplasm (B) (magnification $\times 200$ ).

\section{Discussion and Conclusion}

With the medical technology and equipment evolution, an increasing number of multiple primary neoplasms are being reported. However, MPNs are still rare, accounting for about $0.5 \%$ to $10 \%$ of the incidence of malignant tumors [5]. The etiology of MPNs is still unclear and is generally considered to be the result of multiple carcinogenic factors. Possible etiologies include genetic defects [7,8], medical treatment-related factors [9], environmental factors [10], and unhealthy living habits [11]. Pickled foods contain $\mathrm{N}$-nitrosodimethylamine and other volatile $\mathrm{N}$-nitroso compounds that show mutagenicity and carcinogenicity [12-14]. In this case, the patient had a diet high in salty and pickled foods. She denied using tobacco or alcohol and being exposed to polluted environment. The patient had no history of exposure to ionizing radiation or having undergone chemotherapy. No member in her family was ever diagnosed with neoplasm. Therefore, we suspect that dietary factors are the largest contributor to her MPNs. The treatment of multiple primary neoplasms is fundamentally different from that of metastatic and recurrent cancers [15]. Metastatic and recurrent cancers are mainly treated with nonsurgical palliative care. However, MPNs are mainly treated with surgery-based comprehensive therapy (radical surgery combined with chemotherapy or radiotherapy).

For metachronous MPNs, appropriate treatment options can be selected according to the characteristics of each primary tumor, whereas treatment for simultaneous MPNs must be prioritized for those tumors that have poor prognosis, are directly life-threatening, or have obvious symptoms. The patients who accepted surgerybased comprehensive therapy had a longer survival time than the patients who accepted surgery alone [6]. In the present case, the patient was diagnosed with simultaneous triple primary neoplasms of the ascending colon, transverse colon, and appendix but refused to receive postoperative adjuvant therapy. Therefore, laparoscopic radical extended right hemicolectomy was performed. The prognosis of MPNs is better than that of recurrent and metastatic cancers [16]. In summary, to our knowledge, simultaneous triple primary neoplasms have an extremely low occurrence [17]. The exact etiology of MPNs is still unknown and we suspect that in this case, a diet high in salty and pickled foods was a major contributing factor. Surgery-based comprehensive therapy is the recommended therapy for simultaneous multiple primary neoplasms. However, exact etiologic origin of such MPNs needs to be explored further.

\section{Declarations}

\section{Ethics Approval and Consent to Participate}

This study was approved by the Ethical Review Committee of Guangfu Hospital (Jinhua, Zhejiang, China). The patient signed the informed consent form.

\section{Consent for Publication}

Written informed consent was obtained from the participant for publication of this case report.

\section{Availability of Data and Materials}

All patient data and clinical images are contained in the medical files of Guangfu Hospital in Jinhua. Data is not publicly available due to patient confidentiality concerns but is available from the corresponding author on reasonable request.

\section{Authors Contributions}

All authors participated in the management of the patient in this case report. XYL and GYJ wrote the manuscript; QH and JSL took care of the patient and performed the operation; GYJ, XJZ and YFW revised the manuscript. XJC supervised the entire process. All authors read and approved the final manuscript.

\section{References}

1. Feng Y, Zhong M, Zeng S, Xiao D, Liu Y (2018) Metachronous triple primary neoplasms with primary prostate cancer, lung cancer, and colon cancer: A case report. Medicine (Baltimore) 97(26): e11332.

2. Warren S, Gates $O$ (1932) Multiple primary malignant tumors-a survey of the literature and statistical study. Am J Cancer 16: 358-414.

3. Demandante CG, Troyer DA, Miles TP (2003) Multiple primary malignant neoplasms: case report and a comprehensive review of the literature. American journal of clinical oncology 26(1): 79-83.

4. Koutsopoulos AV, Dambaki KI, Datseris G, Giannikaki E, Froudarakis M, et al. (2005) A novel combination of multiple primary carcinomas: urinary bladder transitional cell carcinoma, prostate adenocarcinoma and small cell lung carcinoma-report of a case and review of the literature. World journal of surgical oncology 3: 51.

5. Zubaidi A (2008) Multiple primary cancers of the colon, rectum, and the thyroid gland. Saudi journal of gastroenterology: official journal of the Saudi Gastroenterology Association 14(4): 202-205.

6. Lv M, Zhang X, Shen Y, Wang F, Yang J, et al. (2017) Clinical analysis and prognosis of synchronous and metachronous multiple primary malignant tumors. Medicine (Baltimore) 96(17): e6799.

7. Cybulski C, Nazarali S, Narod SA (2014) Multiple primary cancers as a guide to heritability. International journal of cancer 135(8): 1756-1763.

8. Takalkar U, Asegaonkar BN, Kodlikeri P, Asegaonkar S, Sharma B, et al. (2013) An elderly woman with triple primary metachronous malignancy: A case report and review of literature. International journal of surgery case reports 4(7): 593-596. 
9. van Eggermond AM, Schaapveld M, Lugtenburg PJ, Krol AD, de Boer JP, et al. (2014) Risk of multiple primary malignancies following treatment of Hodgkin lymphoma. Blood 124(3): 319-327.

10. Burgess P, O'Shea M, Gaskin D, Jonnalagadda R (2010) Coexistence of colonic carcinoma, renal cell carcinoma and gastrointestinal stromal tumour-A case report. International journal of surgery case reports 1(2): 16-18.

11. Berrington de Gonzalez A, Curtis RE, Kry SF, Gilbert E, Lamart S, et al. (2011) Proportion of second cancers attributable to radiotherapy treatment in adults: a cohort study in the US SEER cancer registries. The Lancet Oncology 12(4): 353-360.

12. Scanlan RA (1983) Formation and occurrence of nitrosamines in food Cancer research 43(5 Suppl): 2435-2440.

13. Poirier S, Bouvier G, Malaveille C, Ohshima H, Shao YM, et al. (1989) Volatile nitrosamine levels and genotoxicity of food samples from highrisk areas for nasopharyngeal carcinoma before and after nitrosation. International journal of cancer 44(6):1088-1094.

\section{ISSN: 2574-1241}

DOI: 10.26717.BJSTR.2019.14.002517

Xiujun Cai.Biomed J Sci \& Tech Res

(C) (P) This work is licensed under Creative

Submission Link: https://biomedres.us/submit-manuscript.php
14. Tomita I, Kinae N, Nakamura Y, Takenaka H, Kanamori H, et al. (1984) Mutagenicity of various Japanese foodstuffs treated with nitrite. II. Directly-acting mutagens produced from N-containing compounds in foodstuffs. IARC scientific publications. 1984(57): 33-41.

15. Okada M, Tsubota N, Yoshimura M, Miyamoto Y (1998) Operative approach for multiple primary lung carcinomas. The Journal of thoracic and cardiovascular surgery 115(4): 836-840.

16. Nishimura Y, Komatsu S, Ichikawa D, Okamoto K, Shiozaki A, et al. (2012) The clinical influence of double or multiple primary cancers on the prognosis of patients with gastric cancer. Gan to kagaku ryoho Cancer \& chemotherapy 39(12): 2301-2303.

17. Singh NJ, Tripathy N, Roy P, Manikantan K, Arun P (2016) Simultaneous Triple Primary Head and Neck Malignancies: A Rare Case Report. Head and neck pathology 10(2): 233-236.

\begin{tabular}{ll} 
BIOMEDICAL & Assets of Publishing with us \\
RESEARCHES & - Global archiving of articles \\
\hline ISSN: $2574-1241$ & - Immediate, unrestricted online access \\
\hline
\end{tabular}

